\title{
HCV GENOTYPES AND ITS ASSOCIATION WITH RESPONSE TO TREATMENT
}

Aftab Ahmad Khan ${ }^{a}$, Rizwan Uppal ${ }^{a}$, Naghmi Asif ${ }^{b}$, Gul E Rehanc, Sehrish K. Baloch ${ }^{c}$, Khursheed Ahmad ${ }^{c}$

${ }^{a} \mathrm{Al}-$ Nafees Medical College and Islamabad Diagnostic Center.

${ }^{\mathrm{b}}$ Islamabad Medical \& Dental College and Islamabad Diagnostic Centre.

${ }^{\mathrm{C}}$ Islamabad Diagnostic Center.

\begin{abstract}
:
BACKGROUND \& OBJECTIVE: Hepatitis $C$ is a serious public health problem and is the primary cause of liver cirrhosis, hepatocellular carcinoma (HCC), and numerous end-stage liver disease manifestations. The management of hepatitis $C$ is to preclude liver cirrhosis, lessen the risk of hepatocellular carcinoma or hepatoma, and curing the extra hepatic diseases. Initially, interferon was the cornerstone for treating hepatitis $\mathrm{C}$, but due to its cumbersome complications, route of administration, and limited treatment access, many patients showed noncompliance. New therapies for chronic hepatitis $C$ have been introduced based on direct antiviral effects. Several genotypes of hepatitis $\mathrm{C}$ have been discovered and they are responsive to different antiviral therapies. Our objective was to assess the genotypic distribution of HCV in our local setup and their pattern of response to different combination of anti-viral therapies by assessing the sustained viral response (SVR) after 12 weeks post-treatment. To determine the most prevalent genotype of hepatics $C$ virus in our population and pattern of the response of multiple genotypes to different antiviral regimens.

METHODOLOGY: It is a cross-sectional study conducted for duration of six months and recruited those patients whose polymerase chain reaction (PCR) was found positive for hepatitis $C$ virus at Islamabad Diagnostic Center. We analyzed 100 patients, both children and adults. Patients were assessed for different genotypes and then different combinations of antiviral treatments were administered. Their clinical data, hematological parameters and viral load before and after treatment were also analyzed.
\end{abstract}

RESULTS: In a total of 100 positive hepatitis C virus-infected patients, $55 \%$ were females and $45 \%$ males. The frequencies of genotypes observed were $91 \%, 06 \%$, and $03 \%$ of genotype $3,1 \mathrm{a}$, and $1 \mathrm{~b}$ respectively. 51 out of 91 patients with type 3 genotype, who were on antiviral therapy of sofosbuvir and ribavirin, all of them achieved SVR. 30 out of 91 patients with type 3 genotype were treated with sofosbuvir alone, the percentage of failure to achieve SVR in them was $6.7 \%$. Treatment failure percentage of $10 \%$ was observed when a combination of Interferon (INF) alpha and ribavirin was used in type 3 genotype. Remaining six patients with type $1 \mathrm{a}$ and three patients of type $1 \mathrm{~b}$ genotype achieved SVR with different regimens used.

CONCLUSION: Although the increased load of HCV in our setup is an alarming situation the prevalence of type 3 genotype is a blessing in disguise. The success of sustained viral response after various combinations of direct antiviral therapy and interferon-free treatment is hope for the ultimate cure of the disease and avoidance of debilitating side effects related to interferon.

KEYWORDS: Hepatitis C, Genotypic variations, Sustained viral response.

How to cite this:

doi: https://doi.org/10.37723/jumdc.v11i2.404

Khan AA, Uppal R, Asif N, Rehan G, Baloch SK, Ahmad K. HCV GENOTYPES AND ITS ASSOCIATION WITH RESPONSE TO TREATMENT. jumdc. 2020;11(2):27-33.

doi: https://doi.org/10.37723/jumdc.v11i2.404

This is an Open Access article distributed under the terms of the Creative Commons Attribution License (http://creativecommons.org/licenses/by/4.0), which permits unrestricted use, distribution, and reproduction in any medium, provided the original work is properly cited. 


\section{INTRODUCTION:}

Hepatitis C virus (HCV) infection has been revealed as one of the major health challenges with approximately 200 million infected people worldwide, making about 3.3 percent of the world population and is responsible for almost 350,000 deaths per annum ${ }^{[1]}$. One of the primary reasons for chronic liver disease is $\mathrm{HCV}$ and it is caused by a blood-borne virus, belonging to the family Flaviviridae ${ }^{[2]}$. The current leading risk factor for its transmission is injectable drug abuse and other potential modes of infection include work-related exposure, sexual transmission, unsafe injection use, transfusion of unscreened blood, intranasal cocaine use, tattooing, body piercing, and fetal-maternal transmission ${ }^{[3]}$.

It is a well-recognized global health problem due to its progression to cirrhosis and hepatocellular carcinoma ${ }^{[4]}$. It is usually asymptomatic and chronic disease presenting often with complications. The number of acute infection cases has fallen by more than $80 \%{ }^{[4]}$. However, hepatitis $C$ is yet a major health burden because $60-80 \%$ of infected people progress to chronic infection ${ }^{[5]}$. Hepatocellular carcinoma is the fifth most common malignancy and a major cause of death in patients with chronic HCV infection and is responsible for approximately one million deaths each year ${ }^{[1]}$. Approximately 71 million people are chronically infected with the hepatitis $\mathrm{C}$ virus globally ${ }^{[6]}$ and according to WHO statistics, in South-Asia, Pakistan has the world's second-highest prevalence of hepatitis $C$, and almost 12 million people are suffering from hepatitis $\mathrm{B}$ or $\mathrm{C}^{[7]}$.

On the basis of genomic variability, there are seven major HCV genotypes and 67 subtypes by phylogenetic and sequence analyses of HCV genomes ${ }^{[1]}$. The substantial genetic diversity is more common in genotypes 1-3, distributed globally, while genotypes 4 and 5 limited to the Middle East and Africa, and genotype 6 mostly in south-east Asian countries ${ }^{[2,8,9]}$. Various studies have identified that genotype $3 a$ as the most prevalent HCV genetic variant in Pakistan ${ }^{[2,10]}$. It has been stated that different genotypes have a different course of disease pattern as well as their response to anti-viral therapies is also variable. In patients with chronic HCV genotype $1 \mathrm{~b}$, there is more severe liver disease,

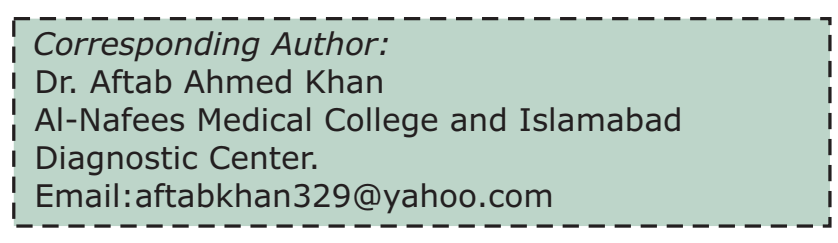

aggressive course and is less effectively treated by interferon than HCV genotype 2 or 3 . Chronicity was also found to be $92 \%$ in patients exposed to HCV genotype $1 \mathrm{~b}$ infection, contrary to $33 \%$ to $50 \%$ in patients exposed to other genotypes ${ }^{[11]}$.

In this era of anti-viral therapy, the treatment of HCV 3 a genotype infections has emerged as the most common treatment-refractory of all the HCV genotypes. A study conducted by Lawitz E et al revealed that there was a lower sustained viral response rate in genotype 3 as compared to genotype 2 with antiviral therapy of sofosbuvir and ribavirin given for 12-16 weeks. Whereas SVR was boosted by using a 12-week course of sofosbuvir and ribavirin plus peginterferon or by prolonging the sofosbuvir plus ribavirin treatment to 24 weeks ${ }^{[12]}$. Various studies have been published on the frequency of HCV genotypes, but very few studies have been conducted to see the association of these genotypes to treatment response. The current project was designed to assess the most prevalent genotype of hepatics $C$ virus in the pakistani population and pattern of response of multiple genotypes to different antiviral regimens.

\section{METHODOLOGY:}

The study was conducted over 6 months at Islamabad Diagnostic Center. The considered population comprised $100 \mathrm{HCV}$ virus-positive patients, detected by polymerase chain reaction (PCR). Abbott RealTime HCV Genotype II was used for discrimination of the Genotypes of Hepatitis $\mathrm{C}$ viral ribonucleic acid (HCV RNA) in human serum/plasma. The study has been approved by the ethical review committee of Islamabad Diagnostic Centre, Islamabad.

After taking informed consent, the patient's blood was collected in an EDTA tube $(3 \mathrm{ml})$ and two gel tubes ( $3 \mathrm{ml}$ in each tube) with a labeled barcode. The patients were selected by consecutive non- probability sampling techniques. The sample in gel tube was 
centrifuged for 15 minutes for separation of serum/plasma.

Initial workup for hematological parameters like hemoglobin, white cell count, platelet count, and alanine aminotransferase was conducted. Along with that, the initial viral load for all the hepatitis $C$ positive patients was also estimated. The patients were administered different combinations of direct-acting antiviral drugs alone or in combination as per prescription by the attending physician. Sofosbuvir alone, INF in combination with ribavirin, and sofosbuvir in combination with ribavirin were used for 12 weeks.

These patients were then assessed for a sustained viral response (SVR) after 12 weeks of treatment. The patient's hematological parameters and viral load were again analyzed post-treatment. Paired T-test was used for any significant difference in those parameters. Data was collected with standardized forms and transferred daily to a server. The descriptive data were analyzed using SPSS version 25.0.

\section{RESULTS:}

In our study, a hundred patients who were found positive for the hepatitis $\mathrm{C}$ virus by Polymerase chain reaction were included. RTPCR for genetic diversification of the hepatitis C virus was performed using Abbott RealTime HCV Genotype II. Patients were also assessed for their pre and post-treatment viral load as well as other parameters like alanine transaminase, hemoglobin, white cell count, and platelets.

The ages of patients varied between 17 years to 81 years with mean age of 47 years. $45 \%$ were males while $55 \%$ were females (Figure-I).

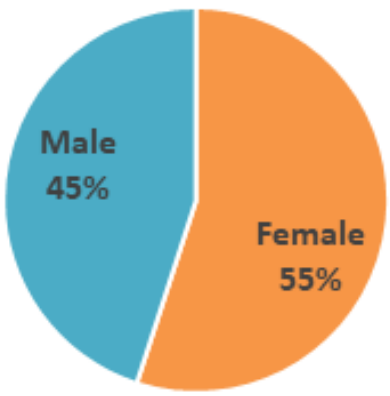

Figure-I: Gender distribution of hepatitis C positive patients.

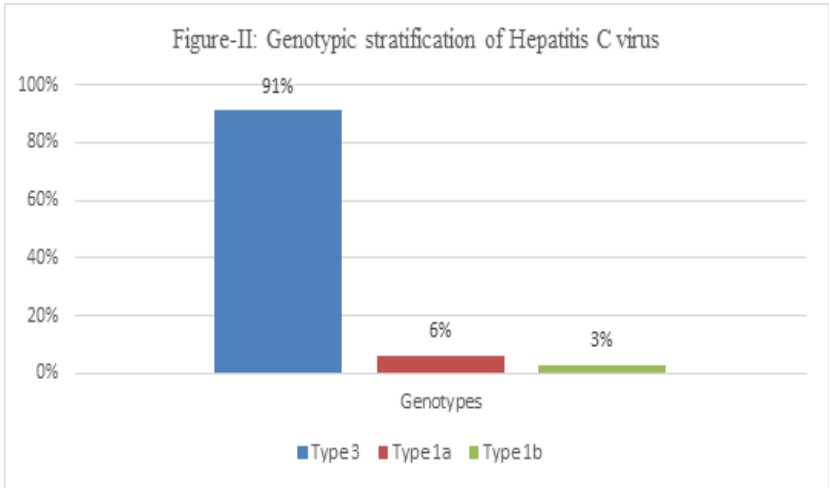

Figure-II: The frequencies of genotypes observed were $91 \%, 06 \%$ and $03 \%$ of genotype $3,1 \mathrm{a}$ and $1 \mathrm{~b}$ respectively.

After genotyping, the patients were put on three different regimens of antiviral therapy as prescribed by the referring physicians. Sofosbuvir alone, INF in combination with ribavirin, and ssofosbuvir and ribavirin were used over the time span of three months. These patients were then assessed for the sustained viral response (SVR) which is defined as the presence of sustained, undetectable HCV RNA levels after at least 12 weeks of completing anti HCV therapy, using a sensitive assay (typically having lower detection limit of $25 \mathrm{IU} / \mathrm{mL}$ ).

By analyzing the three observed genotypes separately, most of the patients were having genotype $3(91 \%)$ and their response varied with different treatment regimens used. They were also stratified according to the viral load before and after the antiviral therapy.

30 out of 91 patients (33\%) with type 3 genotype were treated with sofosbuvir alone. $51(56 \%)$ were treated with sofosbuvir and ribavirin whe reas $10(12 \%)$ were treated with INF and ribavirin regimen (Figure-III).

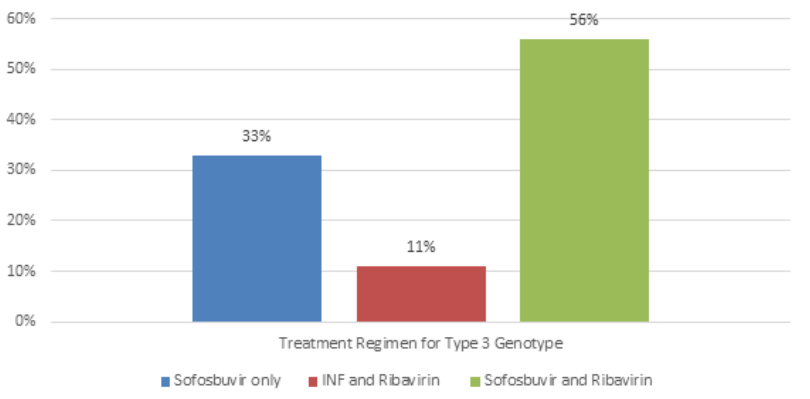

Figure-III: Percentages of various treatment regimens used for type 3 gentoype. 
2 out of 30 patients, who were having a viral load of $>1,000,000$ and treated with Sofosbuvir alone, failed to achieve sustained viral response. This makes the treatment failure rate of Sofosbuvir alone for type 3 genotype of hepatitis C as $6.7 \%$ (Figure-IV).

1 out of the 10 patients, who were also having a viral load of $>1,000,000$ and was treated with a combination of INF and ribavirin, also failed to achieve SVR, making the treatment failure percentage of $10 \%$ (Figure-IV). Whereas all the 51 patients $(100 \%)$ who were treated with sofosbuvir and ribavirin achieved SVR, making it the most effective antiviral therapy in our study (Figure-IV).

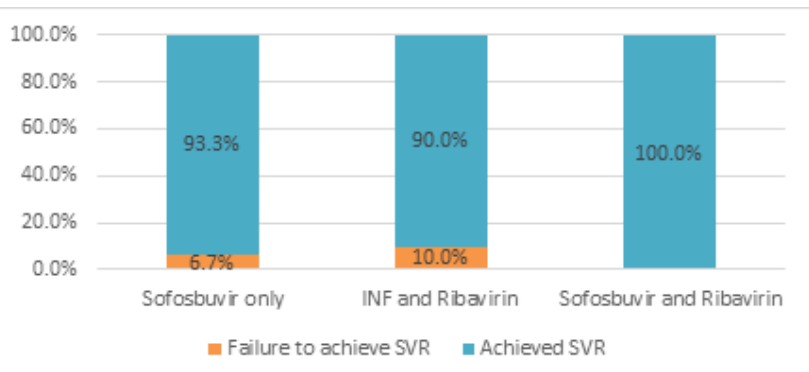

Figure-IV: Response assessment of hepatitis C type 3 genotype to various regimens.

All six patients having HCV genotype 1a achieved SVR with different treatment regimens. Out of these six patients, three were given Sofosbuvir alone, two were given combination of INF and ribavirin and one patient received sofosbuvir and ribavirin (Figure-V).

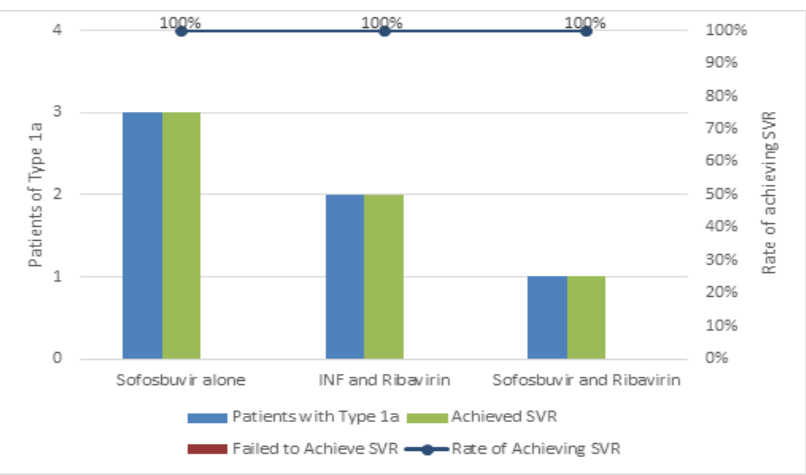

Figure-V: Response assessment of type 1a genotype to various treatment regimens. Similarly all three patients having HCV genotype $1 \mathrm{~b}$ achieved SVR. Two out of these three patients received sofosbuvir and ribavirin whereas one was given combination of INF and ribavirin (Figure-VI).

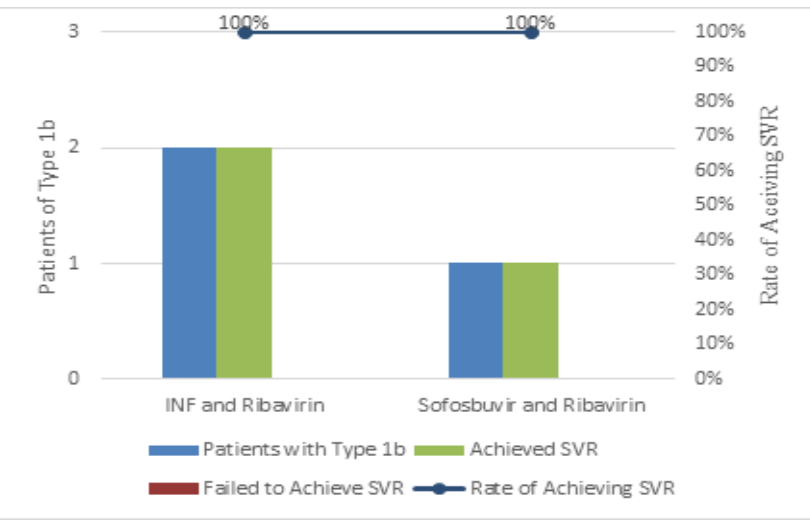

Figure-VI: Response assessment of type 1 b genotype to various treatment regimens.

Pre and post-treatment parameters like alanine transaminase, hemoglobin, white cell count, and platelets were monitored for hepatitis $C$ genotype 3 patients and by using the Paired Ttest only alanine transaminase was found to be significantly altered with treatment (Table-I).

Table-I: Effect of treatment on hematological and biochemical parameters in Genotype 3.

\begin{tabular}{|c|c|c|c|}
\hline Variable & $\begin{array}{c}\text { Before Treatment } \\
\text { (Mean) }\end{array}$ & $\begin{array}{c}\text { After treatment } \\
\text { (Mean) }\end{array}$ & P-value \\
\hline $\mathrm{ALT}$ & 49.3 & 45.0 & 0.05 \\
\hline $\mathrm{Hb}$ & 12.508 & 12.581 & 0.910 \\
\hline Platelets & 213,074 & 223,386 & 0.975 \\
\hline WBC & 9.736 & 9.778 & 0.95 \\
\hline
\end{tabular}




\section{DISCUSSION:}

Viral hepatitis is the most fatal disease worldwide. Hepatitis $C$ virus is a blood-borne virus mostly transmitted through contaminated injection needles, unsafe health care and unscreened blood products. As a result of that, the disease burden is enormous and is prevalent ubiquitously.

Our study included $55 \%$ females and $45 \%$ of males suffering from hepatitis $\mathrm{C}$. The ages of patients varied between 17 years to 81 years with a mean age of 47 years. Studies conducted in developing and underdeveloped countries show no significant statistical difference in the disease burden in terms of age and gender. Contrary to this certain studies conducted in developed countries showed that $\mathrm{HCV}$ infection was more prevalent in their young drug addicts ${ }^{[13]}$.

The frequencies of genotypes of hepatitis $C$ observed were $91 \%, 06 \%$, and $03 \%$ of genotype $3,1 \mathrm{a}$ and $1 \mathrm{~b}$ respectively. A study was conducted in Pakistan for assessing the prevalence of $\mathrm{HCV}$ and its genotypic stratification where it was found out that genotype $3 a$ is the most prevalent one here ${ }^{[2]}$. Contrary to this, globally, genotype 1 is estimated to be approximately $46.2 \%$. HCV genotype 3 is the second most common type and makes up to $30.1 \%$ cases globally, approximately three-quarters of them are in South Asia ${ }^{[14]}$.

After HCV genotyping, the patients were administered different regimens of antiviral therapy. i.e. Sofosbuvir alone, INF in combination with ribavirin, and sofosbuvir in combination with ribavirin for 12 weeks and were then assessed for a sustained viral response (SVR).

30 out of the 91 patients with type 3 genotype were treated with sofosbuvir alone and 2 out of them, having a viral load of $>1,000,000 \mathrm{IU} / \mathrm{mL}$, failed to achieve SVR, making the treatment failure rate of $6.7 \%$. Compared to this, a study was conducted by Jacobson IM et al that showed a failure to achieve sustained virologic response in $22 \%$ in hepatitis $C$ patients of type 3 genotype when treated alone with sofosbuvir [15].

1 out of the 10 patients, treated with a combination of INF and ribavirin and having a viral load of $>1,000,000$, also failed to achieve SVR, making the treatment failure percentage of $10 \%$. In a comprehensive review conducted by Muhammad Umar et al, the SVR to conventional IFN in combination with ribavirin regimen among $\mathrm{HCV}$ patients with genotype $3 a$ is acceptable but if direct acting antivirals (DAA) like Sofosbuvir are available in Pakistan at a cheaper cost then use of DAA is considerably more effective ${ }^{[2]}$. In a study conducted in $\mathrm{HCV}$ 3a patients, the SVR was present in $59 \%$ of patients when treated with a combination of interferon and ribavarin with a viral load of $800,000 \mathrm{IU} / \mathrm{mL}$ pretreatment ${ }^{[16]}$.

Whereas all the remaining 51 patients who were treated with sofosbuvir / ribavirine achieved SVR, making it the most effective antiviral therapy in our study. Another study complimenting our findings show that HCV 3 genotype patients receiving a combination of sofosbuvir and ribavirin, $85 \%$ study population achieved 12 weeks post-treatment SVR ${ }^{[17]}$.

All six patients having HCV 1a genotype and three patients having $\mathrm{HCV} 1 \mathrm{~b}$ genotype achieved SVR with different treatment regimens. A study conducted by Forde AA et al shows that different treatment regimens for genotype $1 \mathrm{HCV}$ infection are highly potent and safe ${ }^{[18]}$. Similarly according to the recommendation published in the journal of Infectious Disease Society of America also reveals that different treatment regimens for genotype $1 \mathrm{~b} \mathrm{HCV}$ infection are favorably effective, safe and SVR is achieved in more than $90 \%$ of patients ${ }^{[19]}$. However, in our study, the number of patients of genotype $1 \mathrm{a}$ and $1 \mathrm{~b}$ is too small to derive a comprehensive conclusion, and therefore a larger study is required for this purpose.

\section{CONCLUSION:}

The significantly high number of HCV 3 genotype in Pakistan is an indication for prevention and control of hepatitis $\mathrm{C}$ infection as the disease response to different treatment regimens is effective enough to achieve sustained viral response. Additionally, the use of antiviral therapy and evolving interferon-free regimens with high sustained virologic response (SVR) rates is the ultimate goal of making the treatment highly effective, inexpensive, safe, 
and tolerable.

\section{CONFLICT OF INTEREST:}

All authors disclose no conflict of interest.

\section{G RANT S UPPORT\& FINANCIAL DISCLOSURES: None.}

\section{REFERENCES:}

1. Kumar A, Rajput MK, Paliwal D, Yadav A, Chhabra R, Singh S. Genotyping \& diagnostic methods for hepatitis $C$ virus: $A$ need of low-resource countries. The Indian journal of medical research. 2018;147(5):445-455. Doi: 10.4103/ijmr. IJMR_1850_16 PMID: 30082568.

2. Umer $M$, Iqbal M. Hepatitis $C$ virus prevalence and genotype distribution in Pakistan: Comprehensive review of recent data. World journal of gastroenterology. 2016 Jan 28;22(4):1684-1700. Doi: 10.3748/wjg.v22.i4.1684 PMID: 26819533.

3. Kim AI, Saab S. Treatment of hepatitis C. The American journal of medicine. 2005 Aug $1 ; 118(8): 808-815$. Doi: $10.1016 / \mathrm{j}$. amjmed.2005.01.073.

4. Wasley A, Alter MJ. Epidemiology of hepatitis $C$ : geographic differences and temporal trends. InSeminars in liver disease 2000;20(1);0001-0016. DOI: 10.1055/s-2000-9506.

5. Modi AA, Liang TJ. Hepatitis C: a clinical review. Oral diseases. 2008;14(1):10-4. Doi:10.1111/j.1601-0825.2007.01419.x.

6. Al Kanaani Z, Mahmud S, Kouyoumjian SP, Abu-Raddad LJ. The epidemiology of hepatitis $C$ virus in Pakistan: systematic review and meta-analyses. Royal Society open science. $2018 ; 5(4): 180257$. Doi: $10.1098 /$ rsos. 180257.

7. World Hepatitis Day 2018 - Daily Times [Internet]. Daily Times. 2019 [cited 30 May 2019].

8. Hajarizadeh B, Grebely J, Dore GJ. Epidemiology and natural history of $\mathrm{HCV}$ infection. Nature reviews Gastroenterology \& Hepatology. 2013;10(9):553-562. Doi:10.1038/nrgastro.2013.107.

9. Li CS, Chan PK, Tang JW. Molecular epidemiology of hepatitis C genotype $6 a$ from patients with chronic hepatitis $C$ from Hong Kong. Journal of medical virology. $2009 ; 81(4): 628-633$. Doi:10.1002/ jmv. 21430.

10. Asian Pacific Association for the Study of the Liver (APASL) Hepatitis C Working Party. Asian Pacific Association for the Study of the Liver consensus statements on the diagnosis, management and treatment of hepatitis C virus infection. Journal of gastroenterology and hepatology. 2007;22(5):615-633. Doi:10.1111/ j.1440-1746.2007.04883.x.

11. Zein NN. Clinical significance of hepatitis C virus genotypes. Clinical microbiology reviews. 2000;13(2):223-235. Doi: 10.1128/CMR.13.2.223.

12. Lawitz E, Mangia A, Wyles D, RodriguezTorres M, Hassanein T, Gordon SC, et al. Sofosbuvir for previously untreated chronic hepatitis C infection. New England Journal of Medicine. 2013 May 16;368(20):18781887. Doi: 10.1056/NEJMoa1214853.

13. Kabir A, Alavian SM, Keyvani H. Distribution of hepatitis $C$ virus genotypes in patients infected by different sources and its correlation with clinical and virological parameters: a preliminary study. Comparative Hepatology. $2006 ; 5(1): 4$. Doi:10.1186/1476-5926-5-4.

14. Shahnazarian $V$, Ramai $D$, Reddy $M$, Mohanty S. Hepatitis $C$ virus genotype 3 : clinical features, current and emerging viral inhibitors, future challenges. Annals of gastroenterology. 2018;31(5):541-551. Doi: 10.20524/aog.2018.0281 PMID: 30174390.

15. Jacobson IM, Gordon SC, Kowdley KV, Yoshida EM, Rodriguez-Torres M, Sulkowski MS, et al. Sofosbuvir for hepatitis C genotype 2 or 3 in patients without treatment options. New England Journal of Medicine. 2013;368(20):1867-1877. Doi: 10.1056/NEJMoa1214854.

16. Thomas DL. Predicting the response to the treatment of hepatitis $\mathrm{C}$ virus infection. Clinical liver disease. 2012;1(2):46-48. Doi: 10.1002/cld.11 PMID: 31186846.

17. Dimitroulopoulos D, Karatapanis S, Kypreos D, Malahias A, Tsamakidis K, Xinopoulos D. The efficacy of 
sofosbuvir/velpatasvir/ribavirin in cirrhotic, genotype $3 a$ HCV patients, previously failures to sofosbuvir/ daclatasvir/ribavirin combination. In Journal OF ViraL Hepatitis. 2018;(25):7171. Doi:10.1111/jvh.66_12923.

18. Forde KA, Bhattacharya D. Treatment of hepatitis $C$ virus (HCV) genotype 1 disease. Current treatment options in infectious diseases. 2017;9(2):262-276. Doi: 10.1007/s40506-017-0124-x PMID: 29805320.

19. hcvguidence.org. Recommendations for Testing, Managing, and Treating Hepatitis C I HCV Guidance [Internet]. Hcvguidelines.org. 2019 [cited 30 May 2019]. Available from: https://www. hcvguidelines.org/.

\section{Authors' Contribution:}

Aftab Ahmad Khan: Substantial contributions to the conception or design of the work or the acquisition, analysis, or interpretation of data for the work.

Rizwan Uppal: Final approval of the version to be published.

Sehrish Baloch: Final approval of the version to be published.

Naghmi Asif: Help in data collection analysis and final draft of study.

Gul E Rehan: Drafting the work or revising it critically for important intellectual content.

Khursheed Ahmad: Drafting the work or revising it critically for important intellectual content.

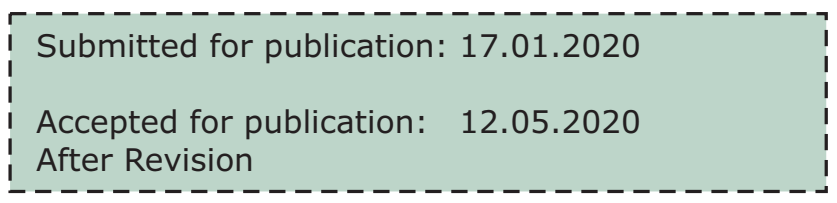

